\title{
A TECHNiQue OF NATURAL Visual LANDMARKS DeteCtion AND DESCRIPTION FOR MOBILE ROBOT COGNiTIVE NAVIGATION
}

\author{
Ekaterina Smirnova, Dmitrii Stepanov, Vladimir Goryunov \\ Central R\&D Institute for Robotics and Technical Cybernetics, Tikhoretsky pr.21, Saint-Petersburg, 194064, Russia
}

\begin{abstract}
A necessary condition for the creation of robotic systems that would be able to replace a human in hazardous conditions is developing means of intelligent control and autonomous navigation providing the possibility of making independent decisions. The article describes a technique of natural visual landmarks detection and description for mobile robot cognitive navigation. The proposed landmarks are built by simultaneous filtering and clustering of a saliency map and a point map that can be achieved by a visual SLAM method like PTAM. As a result the natural landmarks are selected from the scene and described with point clouds and image patches from different point of views. The method significantly improves robot localization robustness (15\% localization error from PTAM is completely removed) and can be used for semantic description of objects in a robot navigation map.
\end{abstract}

Keywords cognitive navigation; mobile robot; computer vision; natural landmarks; point map
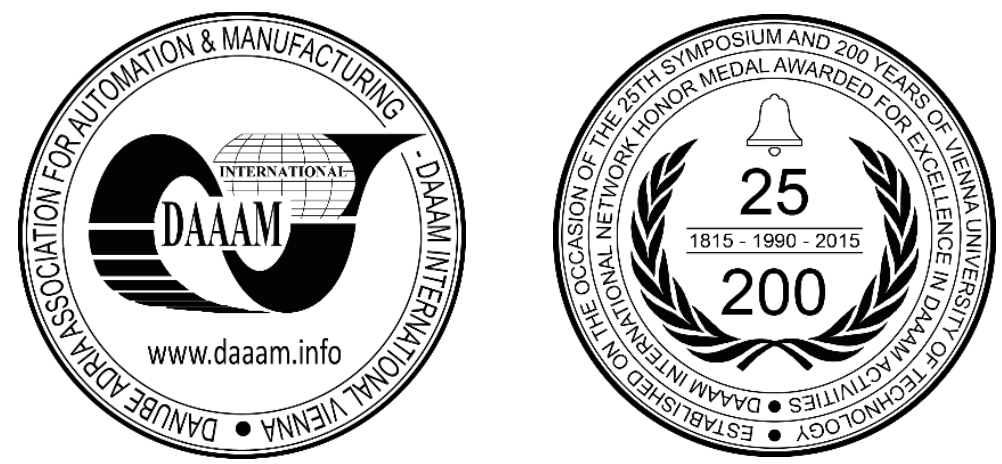

This Publication has to be referred as: Smirnova, E[katerina]; Stepanov, D[mitrii] \& Goryunov, V[ladimir] (2016). A Technique of Natural Visual Landmarks Detection and Description for Mobile Robot Cognitive Navigation, Proceedings of the 26th DAAAM International Symposium, pp.0905-0911, B. Katalinic (Ed.), Published by DAAAM International, ISBN 978-3-902734-07-5, ISSN 1726-9679, Vienna, Austria

DOI: $10.2507 / 26$ th.daaam.proceedings.126 


\section{Introduction}

Typical complex tasks of a mobile robot such as deliveries, exploration of hazardous areas, objects search etc. often assume the robot is outside of a direct control center line of sight. In order to increase the efficiency and reduce the risk of losing of the entire complex it's necessary to propose methods of autonomous execution of such typical tasks with minimal operator intervention.

A necessary condition for the creation of robotic systems that would be able to replace a human in hazardous conditions is developing means of intelligent control and autonomous navigation providing the possibility of making independent decisions. It is necessary to unify the technologies and elements of robotic systems for the purpose of their use:

- $\quad$ as part of multi-purpose robotic complexes;

- $\quad$ selectively on various mobile platforms;

- in a group of mobile robots.

\section{Previous work}

Our own experience and analysis of the related publications revealed that principles of interpreting navigation become one of the most modern and promising approaches to creation of autonomous mobile robots [1]. There are a number of known terrestrial mobile robots [2] that are relatively autonomous at solving of navigation problem in a case of poorly defined obstacles map. Most often their autonomy is limited by the ability of local obstacles avoidance. There are also known navigation complexes based on fusion of information of different physical nature (GPS/GLONASS and inertial navigation system [3]. Their drawback is dependency of an outer source of navigation data (a navigation satellite) and lack of fusion of other available data. A higher level of robot autonomy can be achieved through better use of all available data channels including visual data and a semantic level of data presentation. Such approach would make the robot navigation system closer to the human one, which combine vision, vestibular apparatus, touch and semantical representation of the world in the framework of cognitive maps. Thus the safety and efficiency of complex tasks execution at extreme conditions can be significantly increased. The means for determining of the objects orientations would also allow solving tasks of group robots interaction.

The landmarks can be based on features received from a visual navigation system and from spatial detectors of different physical nature (gamma-visors, gamma-locators, laser and ultrasonic range meters and scanners etc.) [4]. The map which is formed on the basis of the above landmarks is a space of features that describe a sequence of local target designations and observation points, a route between them is overcome without a semantic analysis of the spatial scene. On the route from one observation point to another the local navigation means only solve the problems of safety, local obstacles bypass etc. Using robot sensors and building of topological (non-metric) maps of the operating area also solves the problem of loss of the robot in the absence of external positioning systems.

PTAM (Parallel Tracking and Mapping) [5] is one of the popular approaches that use robotic vision to find landmarks. It is designed to track the camera position and form a point map of a scene without any artificial markers and without any a priori information about the scene. For example, see Fig. 1.

PTAM is designed as two parallel computation threads solving the following tasks:

1. Tracking - tracking of the camera in 3D space;

2. Mapping - construction of map consisting of point clouds, patches and key frames.

To increase the precision (which is necessary for merging of several maps for example) a method of point map filtering and clustering is proposed. A result of the method application is shown on Fig. 2. The result is presented as a projection to the horizontal plane.

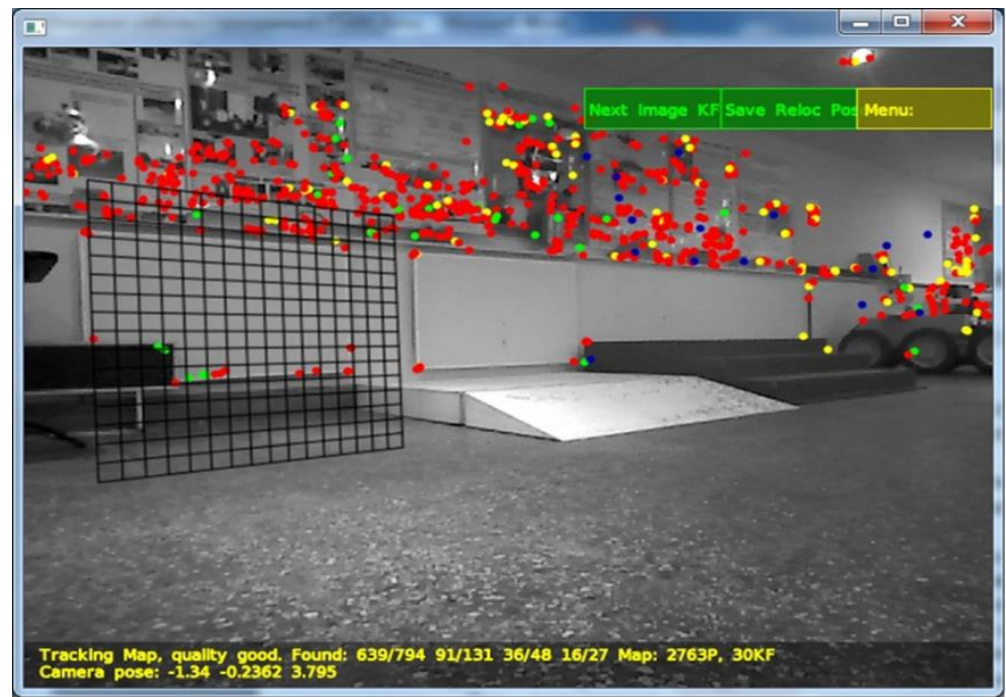

Fig. 1. A sample point map constructed by PTAM. 


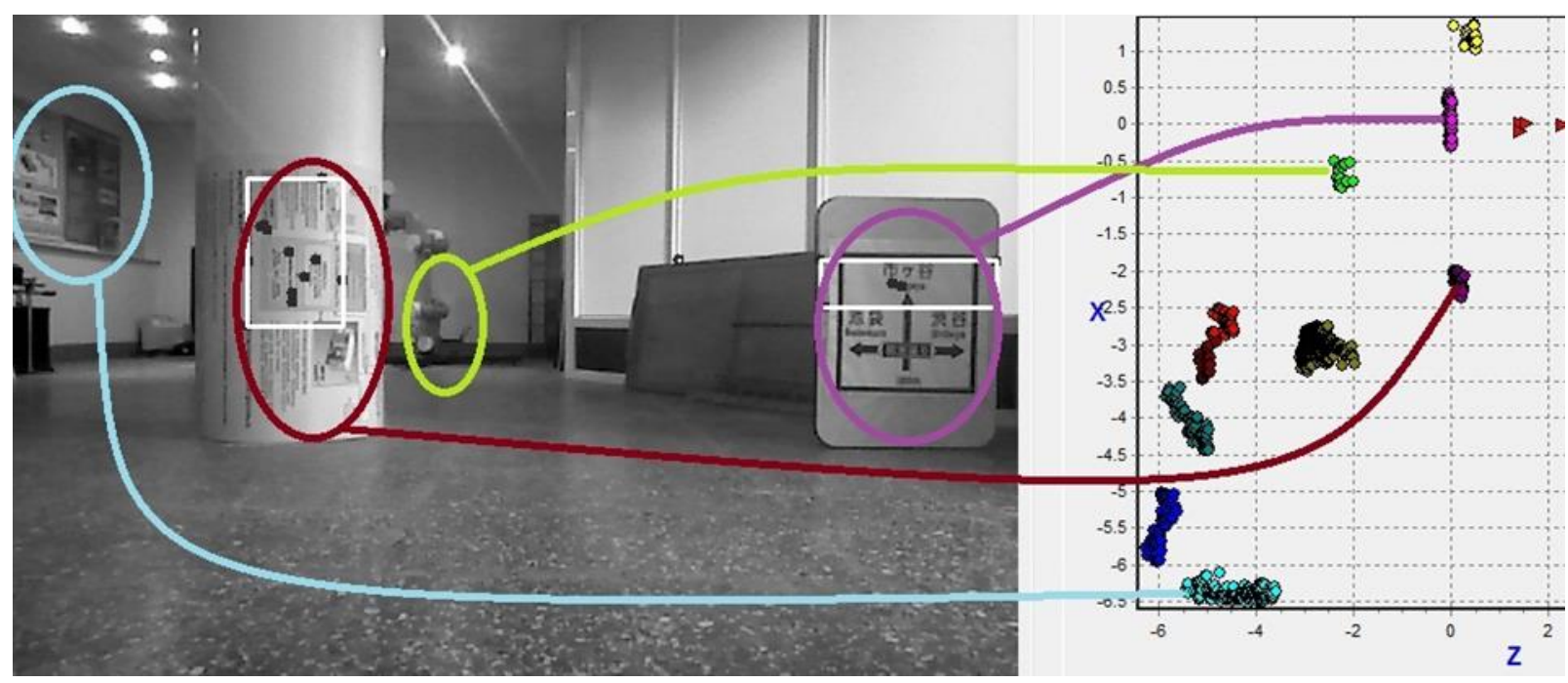

Fig. 2. Point map clustering result.

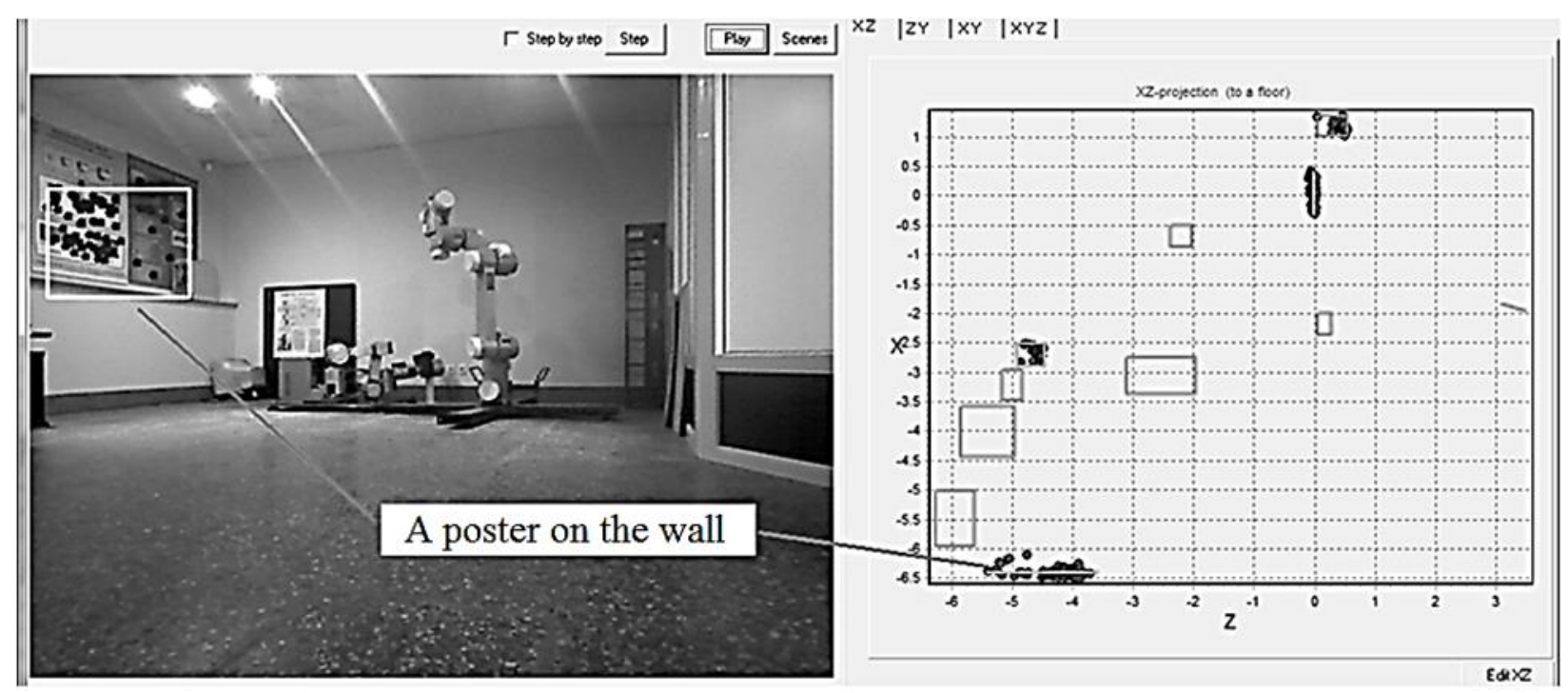

Fig. 3. An example of selection of plane objects from a filtered point map. clusters.

When the clusters are detected from the point map one can do a classification of the objects corresponding to the

In a real scene the most interesting landmarks correspond to plane objects like billboards, elements of building facades etc. The corresponding objects selection method is proposed and the results are demonstrated on the Fig.3.

The PTAM method has several limitations that are significant for navigation of mobile robots:

- A limited practical working area size (10-20 meters);

- An excessive number of points describing the working area (thousands);

- Absence of a topological structure in the map (the map mostly consist of a point cloud);

- Assumption of a static working area, i.e. the objects in the scene should not move, change their sizes or appear;

- The method is sensitive to significant changes in the scene illumination;

- Presence of repeating textures often leads to gross errors of localization and mutual position determination. There is also a popular method TLD (Track Learn Detect) [6], which key feature is parallel detection and tracking of (preselected) natural visual landmarks. According to [7] TLD is still the best available method for real-time visual landmarks tracking. The selection of visual landmarks may be done, using any salience criterion (color, texture, etc.) It also has several limitations from the point of view of the scene analysis task:

- If the landmarks are given from a detection method a landmark can belong to several objects at a time. Sometimes the objects can be placed at a considerable distance from each other;

- The landmarks only have a visual representation, and their mutual spatial position is unknown;

- The landmarks are not related to a spatial map. 


\section{Complex method of search and generation of a list of natural landmarks for mobile robot navigation}

Disadvantages of the above-described methods of landmark detection have a different nature. PTAM method provides a stable construction of the spatial structure, but does not provide a reliable localization due to lack of a common visual picture of the working area. TLD method reliably detects and tracks the visual elements, but is not able to identify steady spatial object on the image. We propose the following method of detection and description of natural landmarks, which significantly improves the accuracy and robustness of the mobile robot navigation.

We propose the following method of detection and description of natural landmarks:

1. While the robot moves the construction of a point map is constantly performed by PTAM with the subsequent natural landmarks detection. Each landmark, its patch and all points related to the patch are assigned an ID. At this step the points are assigned an ID if they lie inside one of the detected landmarks.

Fig. 4 shows a result of projection to the floor plane points belonging to different landmarks. Points with different ID may be grouped together in space. Thus images of the same landmark received from different point of views can be grouped.

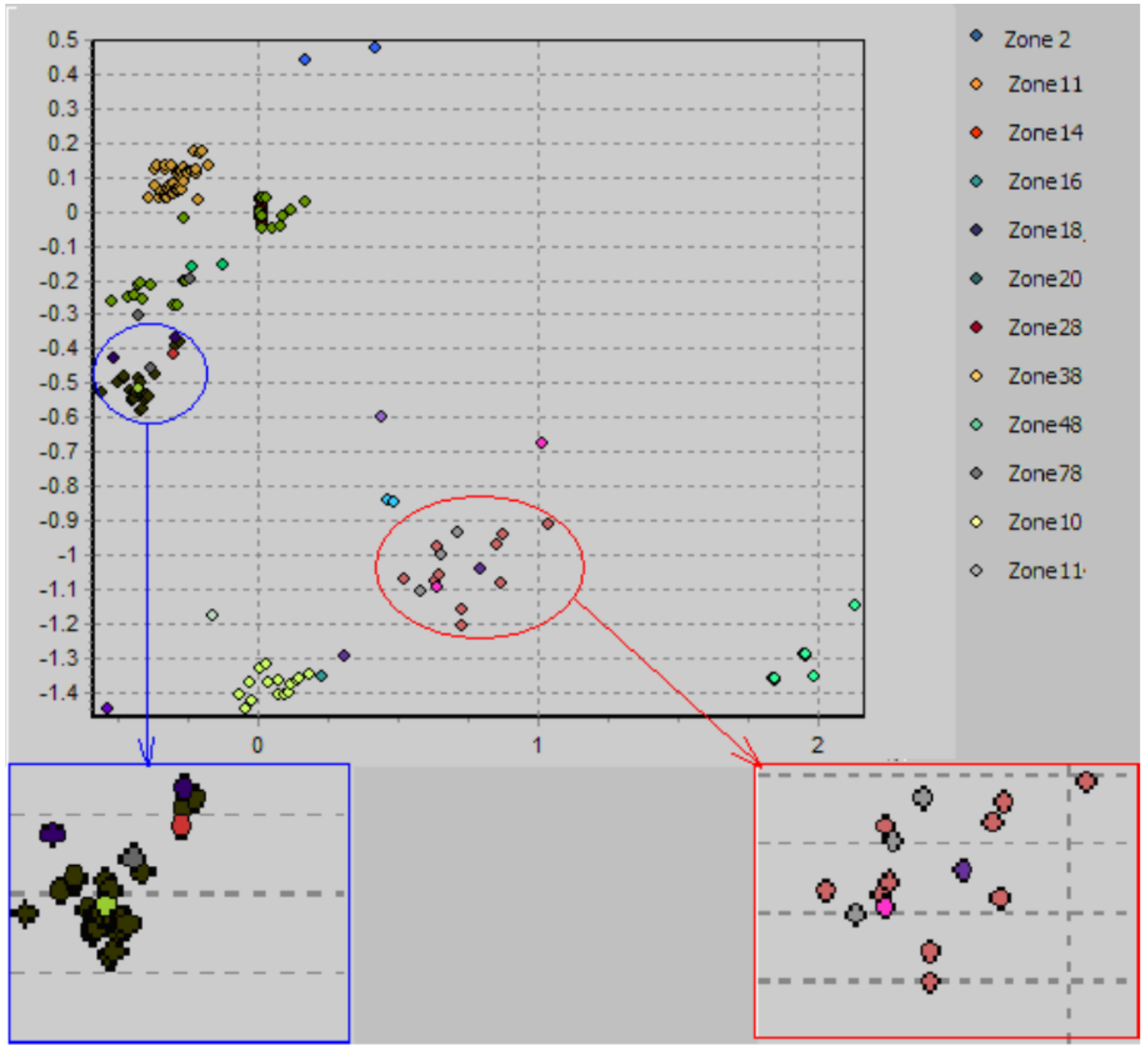

Fig. 4. Points belonging to different landmarks projected to the floor plane.

2. Image patches are assigned to all zones returned by the landmarks detector.

3. Points received at stage 1 . are clustered, and using this clustering the patches from stage 2 . are combined so that a complete set of images of a natural landmark is formed.

4. The formed sets of images of a landmark are post-processed to form a compact landmark description.

\subsection{Detector of natural landmarks}

The detector of the natural landmarks is described with the following sequence, presented at Fig. 5: 


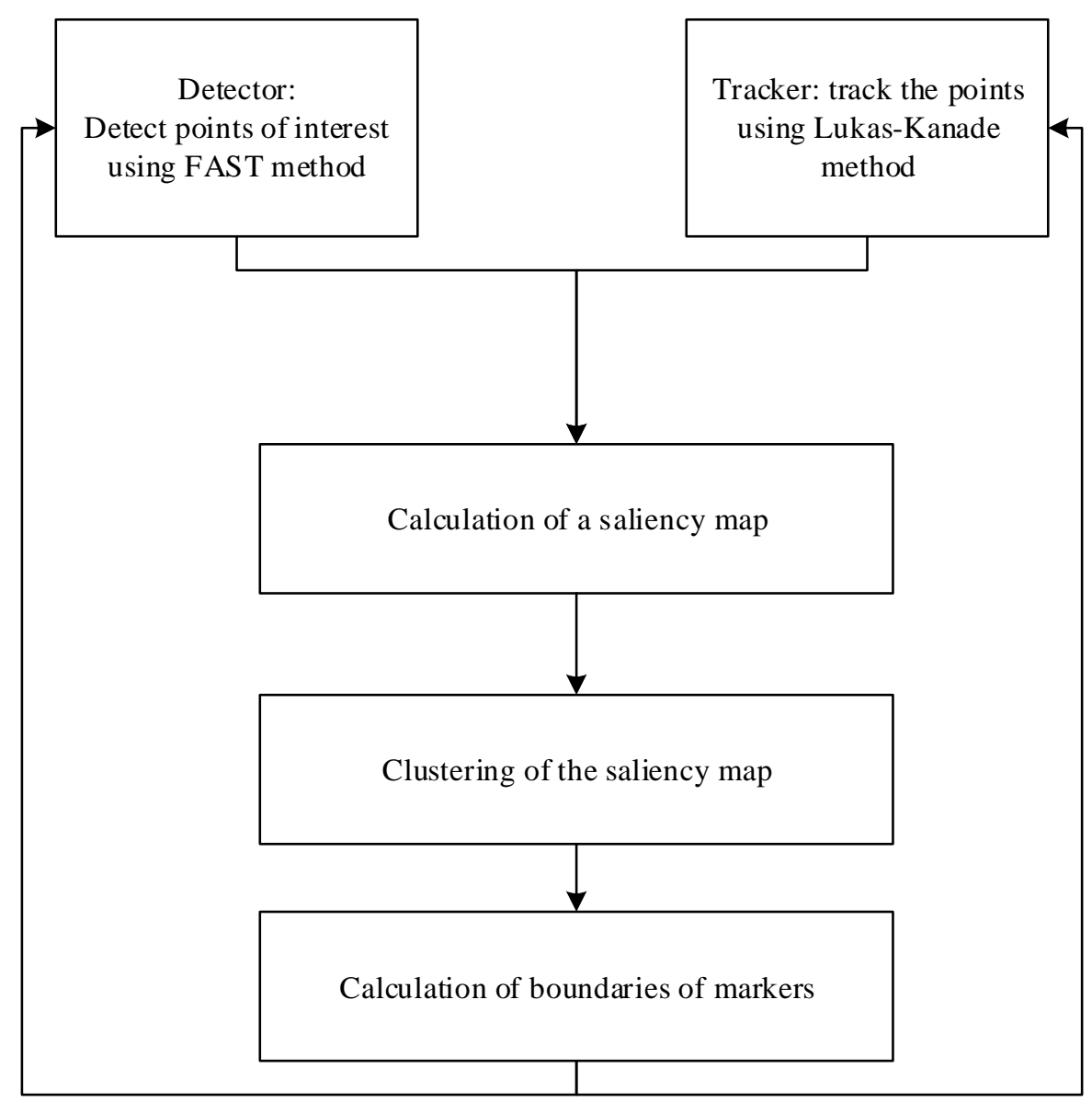

Fig. 5. An algorithm of search and generation of list of natural landmarks.

1. Detection of points of interest is a global procedure initiated for the first and each $\mathrm{N}$-th frame $(\mathrm{N}=5)$ :

1.1 Creation of image pyramid:

- The pyramid size is determined as $\log 2(\min ($ width,height $) / 16)$;

- The image at level " 0 " is initialized with the original frame;

- The image at each following level is calculated by 2-times reduction of the image from the previous level using a Gaussian smooth with sigma $=0.6$ to mimic the retina processing.

1.2 10-points features detector FAST:

- The detector is applied to each pyramid layer (with a threshold 30);

- All the detected points are projected to the original (level " 0 ") image coordinates and added to the global list of points.

2. Tracking of the points of interest is a local procedure initiated at all frames except first and each $\mathrm{N}$-th frames (i.e. where the detection is not initiated):

2.1 The tracker is initialized with the pyramids from the current and the previous frames according to 1.1;

2.2 For each point of interest from the previous frame its new position is calculated based on the pyramid using weighted iterative Lukas-Kanade tracker. The points with their new coordinates are added to the resulting list:

- For each pyramid level (top down) optical flow is calculated using the points processed at the previous level. The calculation is done using the weighted iterative Lucas-Kanade tracker with maximum of 4 iterations and precision of 0.1. The least squares method window size is $7 \times 7$. The weights are logarithmic.

3. Calculation of saliency map is initiated at each frame

3.1 Initialization of the saliency map.

- The saliency map is an image that is 10 times less than the original frame. It's initialized with zeros;

- For each point of interest detected or tracked at the current frame the saliency map is updated. A value of each point of the saliency map that is in a (scale $\times$ scale +1$)$ window around the current point of interest is increased by scale. Here scale is the level of the pyramid where the point is detected.

3.2 Saliency map normalization

- Values of the map are sorted;

- Five minimal and five maximal values are discarded as outliers;

- The left values are normalized to $[0 ; 1]$ range.

3.3 Saliency map threshold

- The values bigger that a threshold (0.3) set to 1 , others - to 0 . 
4. Saliency map clustering is initiated at each frame

4.1 Clear the clusters list;

4.2 Process each non-zero point of the saliency map

- If the clusters list is empty, a new cluster consisting of the current point is created

- Else find the nearest cluster using Euclidean distance. If the minimal distance is less than a threshold (3.0) then the current point is associated to the cluster, else a new cluster is created.

5. Calculation of marker boundaries by the clusters is initiated at each frame.

5.1 Clusters with less than 8 points are discarded as unreliable;

5.2 Calculation of the minimal bounding box describing most cluster points:

- The points of the cluster are sorted and the minimal bounding box containing all the points is estimated;

- The boundaries are corrected by reducing the box size by $40 \%$ thus discarding the outliers;

- The bounding box coordinates are converted to the initial frame coordinate system;

- Too small boxes (size in any dimension $x$ or $y$ is less than 7) are discarded.

5.3 The resulting bounding boxes are the boundaries of the landmarks.

\subsection{The method of combination of patches of landmarks}

The method of combination of patches of landmarks a posteriori reduces the number of image patches associated to a landmark by discarding of images that are similar to other patches associated to the same landmark:

1. The input for the algorithm is a set of patches associated to a landmark received with the above method.

2. Distances between all the pairs of the patches are calculated as follows:

2.1 The distance is infinite if the intersection between mean color HSV-histograms of the two patches is less than 0.3. Such pairs are discarded from the future analysis.

2.2 The normalized cross-correlation coefficient (NCC) is calculated;

2.3 If the NCC is less than 0.4 then the distance is set to infinite, else it's set to $1 / \mathrm{NCC}$

3. The set of patches is clustered according to the distances.

4. Mean color HSV histograms are then recalculated for each cluster resulted from the previous step.

5. The process is repeated until all the similar patches are combined.

The distinctive feature of the complex method is that, during the robot movement two procedures are executed in parallel:

- Construction of a spatial point map by PTAM and clustering of point maps;

- $\quad$ Tracking and storage of visual patches of relevant point clusters by TLD.

So we get a set of dynamic images of the same spatial object (visual natural landmark), obtained from different points of view.

To reduce the number of stored images, we use a posteriori analysis and exclude too similar images. We use the normalized cross-correlation (NCC) as a measure of similarity.

\section{Experiments}

Test runs of the mobile robot were carried out in two different work zones shown in Figure 6.
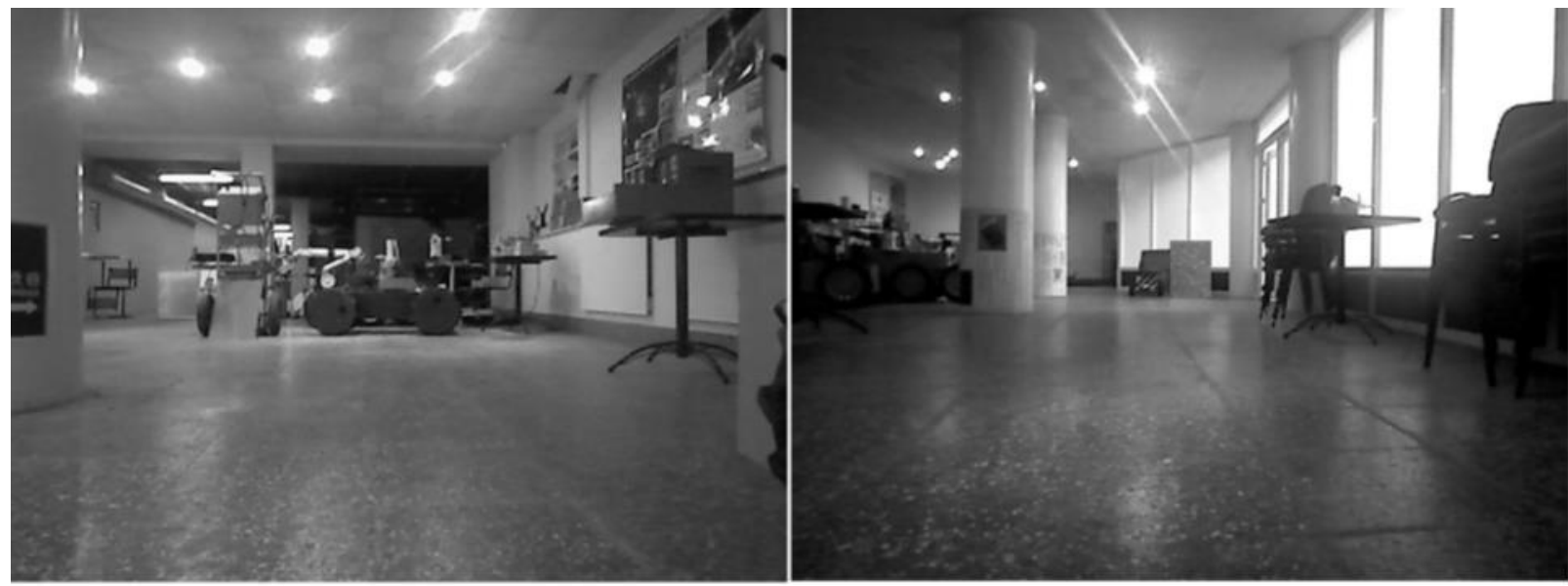

Fig. 6. Examples of a mobile robot working zones.

Sets of visual patches corresponding to the objects of the work zones, which can be used as landmarks (e.g. posters, compact objects, etc.), were created for both zones. Examples of such sets are shown in Figure 7. 


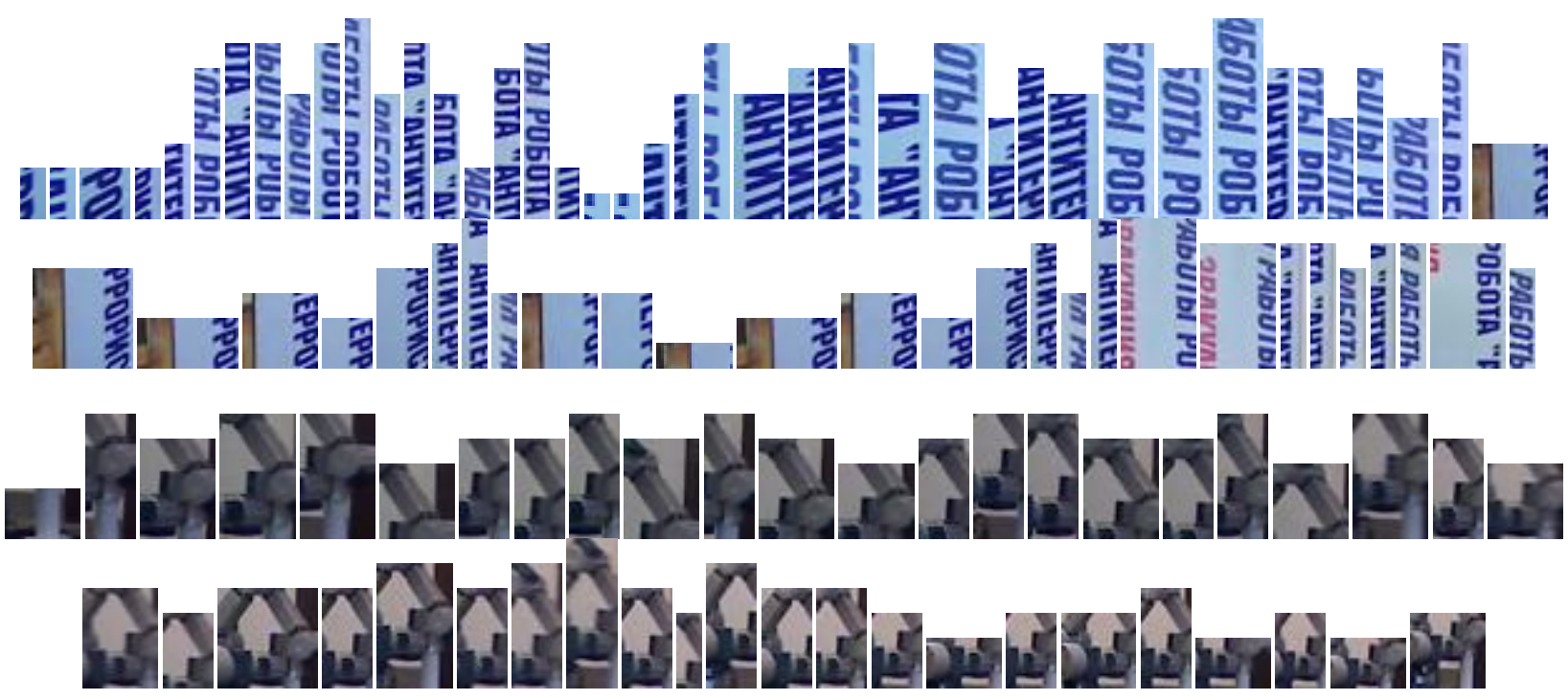

Fig. 7. Examples of sets of visual patches, corresponding to the landmarks of mobile robot work zones. (a - poster b design element)

Using the PTAM algorithm to natural landmarks detection resulted in a $15 \%$ robot localization error. Checking the robot position against the landmarks completely removed the localization error.

The landmarks detection process uses no metric measurements. Thus the landmarks map can be successfully used for cognitive navigation in the space of these landmarks without referencing a particular coordinate system.

\section{Conclusion}

The described combined algorithm of selection of natural visual landmarks from a mobile robot working area significantly increases robustness of robot localization. The natural landmarks detected with the proposed method correspond to compact special objects. This result is typical for the traditional cartography. Thus such landmarks can be used to:

1. Form reliable maps of a working area at a scale that is convenient for robot navigation;

2. Describe robot map objects at a semantic level using form, color and other attributes;

3. Implement navigation of a mobile robot in a topological space of natural landmarks.

The studies and obtained results allow outlining the future research directions:

- implementation of fusion of metrical information and visual landmarks space with the use of sensory information from an inertial navigation system and a multichannel robotic vision system;

- development of a physical model (mockup) of such system;

- detailed experimental studies of the proposed methods using the mockup.

We're also planning to migrate our combined algorithm to LSD-SLAM (Large-Scale Direct Monocular SLAM) method presented in [8]. It significantly outperforms PTAM in terms of density of the generated point maps providing our landmarks selection algorithm with better initial data.

\section{References}

[1] A.A. Kirilchenko, A.K. Platonov, S.M. Sokolov, Theoretical aspects of organization of interpreting navigation of a mobile robot. // M.: Preprint by Keldysh Institute of Applied Mathematics, 2002, Issue 5, 40 p.

[2] I.V. Rubtsov, The state and prospects of development of national terrastrial military and special robotics// Izvestiya of SFedU. Engineering Sciences, 2013. Issue 3(140), pp.14-21.

[3] A.P. Mezentsev, V.M. Achildiev, A.I. Tereshkin, A.N. Naumov, A.V. Shishlov, V.Y. Yurov, Universal navigation device for motion control based on micromechanical sensors and an unified integrated strapdown inertial navigation system for this device. Patent RU № 2263282, 27.10.2005.

[4] A.V. Lopota, A.V. Bakhshiev, V.V. Goryunov, M.N. Plavinskii, E.Y. Smirnova, Prospects of the use of interpreting navigation and fusion in mobile robots control systems. Defense Engineering Problems. Series 16. Issue 11-12/2014. pp. 104-110.

[5] G. Klein, D. Murray, Parallel tracking and mapping for small ar workspaces. In proc. International symposium on mixed and augmented reality, ISMAR'07, Nara.

[6] Z. Kalal, K. Mikolajczyk, J. Matas, Tracking-learning-detection. Pattern Analysis and Machine Intelligence, IEEE Transactions on, 34(7), pp. 1409-1422.

[7] D. Wang, H. Lu, and M. Yang, Online object tracking with sparse prototypes. IEEE Transactions on Image Processing, 22(1), 2013, pp. 2411-2418.

[8] J. Engel , T. Schöps, D. Cremers, LSD-SLAM: Large-scale direct monocular SLAM. In Computer Vision-ECCV 2014, pp. 834-849. Springer International Publishing. 